\title{
Influência da geometria do pino da ferramenta de soldagem nas propriedades mecânicas e tensões residuais de juntas soldadas de ligas de alumínio 5052-O pelo processo FSW
}

\author{
Influence of the welding tool geometry on the mechanical properties and residual \\ stresses of the 5052-O aluminum alloys welded joints by the FSW process
}

Eduardo Bruno Souza de Oliveira1', Déborah Brito Colaço², Mattheus Apolinário Ribeiro², Jefferson Segundo de Lima",

Theophilo Moura Maciel², Raphael Henrique Falcão de Melo ${ }^{1}$

\section{RESUMO}

Ligas de alumínio possuem excelentes características e propriedades mecânicas. No entanto, a soldagem desses materiais através de processos convencionais ao arco elétrico resulta em uma degradação excessiva destas propriedades, afetando diretamente sua vida útil. Neste contexto, os processos de soldagem por fricção têm recebido atenção nos últimos anos, principalmente devido à minimização do aporte de calor fornecido durante o processo, não atingindo o ponto de fusão do material. Este trabalho tem por objetivo analisar diferentes perfis de pino da ferramenta de soldagem por FSW e sua influência nas propriedades mecânicas das juntas soldadas. Para tanto, 12 geometrias de ferramentas foram projetadas, fabricadas e testadas. Ensaios mecânicos de dobramento de raiz a $60^{\circ}$, tração uniaxial, determinação do perfil de dureza e de tensões residuais das juntas soldadas foram empregados para caracterização e análise das propriedades. Observou-se uniformidade tanto nos perfis de dureza como nos de tensões residuais. Os ensaios de tração uniaxial e dobramento de raiz indicaram que a ferramenta 1 , com pino em formato de tronco de cone e com rosca, obteve valores de resistência mecânica inferiores às demais, sendo as ferramentas 4 e 9, cilíndricas com e sem rosca, respectivamente, as que proporcionaram as melhores propriedades mecânicas.

Palavras-chave: Friction stir welding, Liga de alumínio, Geometria da ferramenta.

\begin{abstract}
Aluminum alloys have excellent characteristics and mechanical properties. However, welding these materials through conventional arc welding process results in an excessive degradation of the original properties of the base metal, directly affecting the joint lifespan. In this context, friction stir welding process have received attention in the last years, mainly due to the minimization of the heat supply provided during the process, not reaching the material melting point. This work aims to analyze different pin profiles of the welding tool by FSW and their influence on the mechanical properties of welded joints. To that end, 12 welding tool geometries were designed, manufactured and tested. Mechanical tests of root bending at $60^{\circ}$, uniaxial tensile test, hardness profile measurement and residual stress profiles of the weld joints were used to characterize and analyze the resulting properties. It was observed in both hardness and residual stress profiles. Uniaxial tensile tests and root bending indicated that the tool 1, with truncated cone shape pin and screw thread, obtained the lowest values of mechanical resistance, and tools 4 and 9 , cylindrical with and without screw thread in the pin, respectively, the ones that provided the best mechanical properties.
\end{abstract}

Keywords: Friction stir welding, Aluminum alloys, Tool geometry.

\footnotetext{
${ }^{1}$ Instituto Federal de Educação, Ciência e Tecnologia da Paraíba - Unidade Acadêmica de Indústria - Cajazeiras/PB - Brasil. ¿Universidade Federal de Campina Grande - Departamento de Engenharia Mecânica - Campina Grande/PB - Brasil. Autor correspondente: Raphael Henrique Falcão de Melo | Instituto Federal de Educação, Ciência e Tecnologia da Paraíba - Unidade Acadêmica de Indústria | Rua José Dantas Nobres, 131 | CEP 58.900-000 - Cajazeiras/PB - Brasil | E-mail: raphael.melo@ifpb.edu.br Recebido: 01 Mar. 2018 | Aprovado: 26 Abr. 2018
} 


\section{INTRODUÇÃO}

A crescente demanda por produtos de maior qualidade e durabilidade atrelados à redução de consumo e preservação do meio ambiente, faz com que a utilização do alumínio venha experimentando um constante crescimento nos setores da indústria automotiva, naval e aeronáutica, visto que a combinação de baixa densidade, alta resistência à corrosão, boa tenacidade, excelente conformabilidade e reciclabilidade garantem sua utilização em escala industrial. Além disso, alumínio pode ser combinado com uma larga gama de outros elementos de liga, permitindo a otimização de propriedades, ampliando ainda mais sua aplicabilidade ${ }^{1-3}$.

Dentre os diversos tipos de ligas de alumínio, as ligas de $\mathrm{Al}-\mathrm{Mg}$ (série $5 \mathrm{XXX}$ ) são as mais utilizadas em escala industrial por oferecer excelente relação entre peso e propriedades mecânicas, elevada resistência à corrosão e boa soldabilidade ${ }^{4}$. Essas ligas são de uso geral para aplicações de moderada resistência mecânica que necessitam de resistência à corrosão, como tanques de armazenamento e vasos de pressão, bastante utilizados nas indústrias petroquímicas e em estruturas navais. A soldagem é o principal processo de fabricação empregado na confecção destes componentes.

No entanto, a soldagem de ligas de alumínio por métodos convencionais, tais como soldagem ao arco elétrico, onde ocorre a fusão do metal de base, requer diversas precauções a fim de evitar a perda das propriedades mecânicas devido ao coalescimento dos grãos, formação de estruturas brutas de solidificação, geração de vazios, distorção após a soldagem, tensões residuais de fabricação, entre outros ${ }^{3,5}$. Alternativas que visam contornar tais dificuldades impostas por processos tradicionalmente utilizados, como Gas Metal Arc Welding (GMAW) e o Gas Tungsten Arc Welding (GTAW), vêm motivando o desenvolvimento de novos métodos de união, incentivando a aplicação da soldagem por atrito de forma mais intensa, onde a união de peças ocorre sem fusão ${ }^{3,6}$.

Logo, o processo denominado Friction Stir Welding (FSW) apresenta-se como uma alternativa à soldagem por fusão ao arco elétrico tradicional, que pode propiciar precipitação de fases frágeis e outros defeitos relacionados a solidificação, como porosidades e trincas a quente, permitindo a obtenção de microestruturas com propriedades mecânicas, em muitos casos, superiores às obtidas por processos convencionais ${ }^{7}$. Este processo de soldagem é interessante para as séries de ligas de alumínio termicamente tratáveis, que têm sua resistência mecânica aumentada pela formação de precipitados de fases, e baixa soldabilidade por processos de soldagem por fusão, como ligas das séries $2 \mathrm{XXX}$ e $7 \mathrm{XXX}^{8,9}$. Por outro lado, o processo FSW também é interessante para as séries de ligas trabalháveis onde o aumento de resistência mecânica é obtido pela deformação mecânica a frio, tais como as ligas $1 \mathrm{XXX}, 3 \mathrm{XXX}, 4 \mathrm{XXX}$ e 5XXX, em função de benefícios como aumento da resistência à fadiga e menor nível de tensão residual ${ }^{6,10,11}$.
Desenvolvido e patenteado pelo The Welding Institute (TWI), em 1991, o FSW possui diversas variáveis de processo que influenciam no desempenho da junta soldada e, por ser uma técnica de soldagem relativamente nova, estudos prospectivos da utilização desse método e a influência de seus parâmetros de processo nas propriedades mecânicas e magnitude das tensões residuais de fabricação são necessários, tanto para compreender o mecanismo de geração destas tensões, como para assimilar as aplicações industriais dessas juntas soldadas ${ }^{6,7,12}$.

As tensões residuais são acumuladas nas juntas soldadas como consequência da soldagem, podendo modificar a resistência mecânica das juntas, como por exemplo, a vida em fadiga, bem como a resistência à corrosão ${ }^{13}$. As tensões residuais são resultantes de transformações microestruturais não homogêneas que implicam em alterações volumétricas, devido ao aporte de calor extremamente intenso e localizado dos processos de soldagem ao arco elétrico, resultando em comportamento diferente em cada região da junta, com temperaturas diferentes, taxas de resfriamento diferentes e, portanto, microestruturas diferentes ${ }^{14}$. Isto não se verifica em processos de soldagem no estado sólido, como o FSW, com a mesma intensidade.

O processo FSW consiste em uma ferramenta rotativa de elevada resistência mecânica e com um perfil especial, que é inserida no material e avança de modo contínuo e, mediante o atrito com as peças a serem soldadas, gera calor, promovendo a mistura mecânica dos metais envolvidos, confeccionado uma junta soldada de alta qualidade ${ }^{3,15}$. Além disso, outro aspecto importante do processo FSW é que para sua realização não é necessário nenhum material de adição, portanto o metal de base pode ser soldado sem maiores preocupações correlacionadas à compatibilidade de sua composição.

A ferramenta de soldagem é composta, fundamentalmente, por pino, ombro e pelo corpo da ferramenta, e tem duas funções preliminares: aquecimento localizado e promoção do fluxo do metal na zona misturada. A geometria da ferramenta é um dos parâmetros mais influentes de todo o processo FSW. O pino, através de uma força axial aplicada, penetra no material a ser soldado, promovendo aquecimento e severa deformação devido ao movimento de rotação. O ombro é responsável por não deixar o material misturado se espalhar. A ferramenta gira rapidamente entre duas chapas posicionadas de topo, forçando o material a fluir e destruir a interface de separação entre elas, promovendo a união ${ }^{16,17}$. Embora o processo FSW tenha merecido destaque, sendo objeto de estudo em diversos centros de pesquisa tecnológica no exterior, tem se observado poucos trabalhos realizados inteiramente no Brasil, especialmente no que se trata de soldagem de ligas de alumínio pelo processo FSW ${ }^{3,6,18-23}$.

Nesse contexto, este trabalho tem por objetivo avaliar a influência da geometria de diferentes pinos de ferramenta de soldagem nas propriedades mecânicas, bem como na magnitude das tensões residuais de juntas soldadas da liga de alumínio AA 5052 - O utilizando o processo FSW. 


\section{MATERIAIS E MÉTODOS}

Neste trabalho foram utilizadas chapas de alumínio da liga 5052-O com 4,76 mm de espessura. A composição química das chapas, segundo o fabricante, é apresentada na Tabela 1.

Tabela 1: Composição da liga 5052 (\%).

\begin{tabular}{c|c|c|c|c|c|c} 
Mg & Fe & $\mathrm{Cu}$ & $\mathrm{Mn}$ & $\mathrm{Cr}$ & $\mathrm{Zn}$ & $\mathrm{Al}$ \\
\hline 2,2 & 0,4 & 0,1 & 0,1 & 0,25 & 0,1 & Balanço \\
\hline
\end{tabular}

A fim de averiguar a influência da geometria da ferramenta na resistência mecânica e na magnitude das tensões residuais, foram fabricados doze perfis de ferramentas diferentes, como pode ser observado na Fig. 1 e na Tabela 2. As ferramentas foram usinadas em aço ao carbono ABNT 1020, submetidas a cementação em caixa a $925^{\circ} \mathrm{C}$ por $4 \mathrm{~h}$, seguida de têmpera em água agitada, a fim de aumentar a dureza e resistência ao desgaste do pino e ombro da ferramenta.

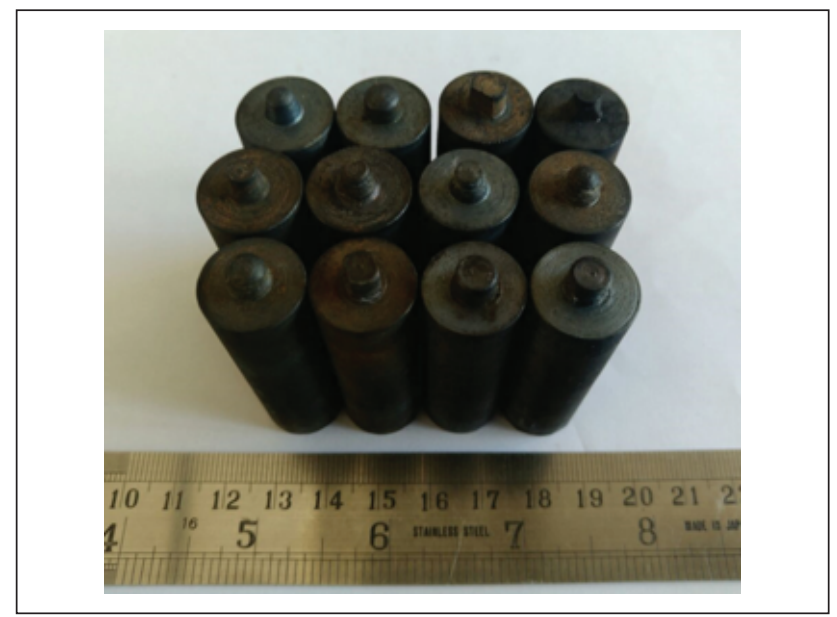

Figura 1: Ferramentas de soldagem confeccionadas em 12 perfis diferentes de pinos.

As juntas soldadas foram confeccionadas utilizando uma fresadora universal automática modelo FU-300 da Diplomat, onde é possível ajustar inclinação do cabeçote que suporta a ferramenta de soldagem, rotação e velocidade de translado. A inclinação da ferramenta de soldagem foi mantida em $2^{\circ}$, a rotação utilizada foi de $1750 \mathrm{rpm}$ e a velocidade de soldagem foi de $118 \mathrm{~mm} / \mathrm{min}$. Na Fig. 2 apresentam-se as dimensões da junta soldada. $O$ parâmetro de controle sob avaliação é a geometria do pino da ferramenta de soldagem.

Os corpos de prova para os ensaios de tração e dobramento a $60^{\circ}$ são apresentados nas Figs. 3 e 4, respectivamente. Esses ensaios foram realizados em uma máquina universal de ensaios da Instron, modelo 5582. Nos ensaios de tração foram utilizados três corpos de prova para cada condição soldada, sendo aplicada uma taxa de deslocamento de $0,05 \mathrm{~mm} / \mathrm{min}$ até a ruptura dos
Tabela 2: Perfis da geometria do pino da ferramenta.

\begin{tabular}{|c|c|c|}
\hline Ferramenta & Imagem & Nome \\
\hline 1 & & Cônico de ponta reta \\
\hline 2 & & $\begin{array}{l}\text { Rosqueado cônico de } \\
\text { ponta arredondada }\end{array}$ \\
\hline 3 & & $\begin{array}{c}\text { Rosqueado cônico } \\
\text { facetado de ponta reta }\end{array}$ \\
\hline 4 & & $\begin{array}{l}\text { Rosqueado cilíndrico de } \\
\text { ponta reta }\end{array}$ \\
\hline 5 & & $\begin{array}{l}\text { Rosqueado cilíndrico de } \\
\text { ponta arredondada }\end{array}$ \\
\hline 6 & & Rosqueado cônico de \\
\hline 7 & & $\begin{array}{l}\text { Cônico de ponta } \\
\text { arredondada }\end{array}$ \\
\hline 8 & & $\begin{array}{l}\text { Rosqueado cônico } \\
\text { facetado de ponta } \\
\text { arredondada }\end{array}$ \\
\hline 9 & & Cilíndrico de ponta reta \\
\hline 10 & & Cilíndrico facetado de \\
\hline 11 & & $\begin{array}{l}\text { Cônico facetado de ponta } \\
\text { reta }\end{array}$ \\
\hline 12 & & Cilíndrico de ponta \\
\hline
\end{tabular}


corpos de prova. Já nos ensaios de dobramento foram utilizados dois corpos de prova para cada perfil de ferramenta, sendo os ensaios realizados voltados para a raiz da junta.

Os ensaios de dureza utilizando um microindentador Vickers (HV) foram executados aplicando-se uma carga de 50 gramasforça (gf) por $15 \mathrm{~s}$, com espaçamento entre as impressões de $200 \mu \mathrm{m}$, conforme Fig. 5. Em cada amostra foram realizadas medidas ao longo de apenas uma linha horizontal transversal ao cordão de solda utilizando um microdurômetro digital FM-700 da FutureTech.

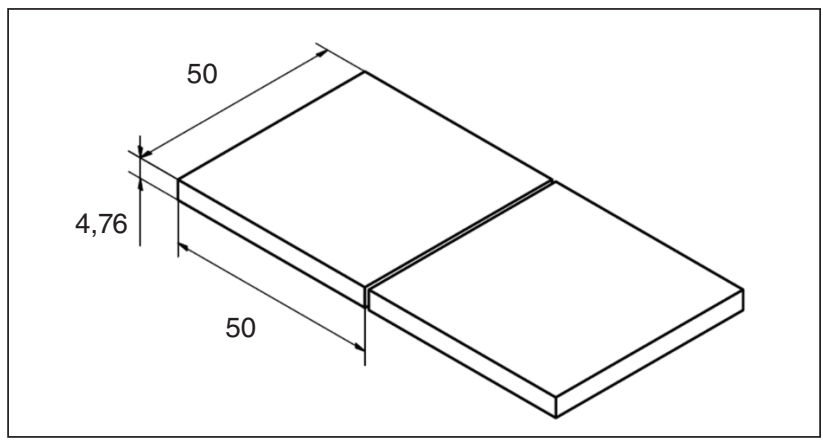

Figura 2: Esquema da junta de topo empregada neste trabalho (dimensões em mm).

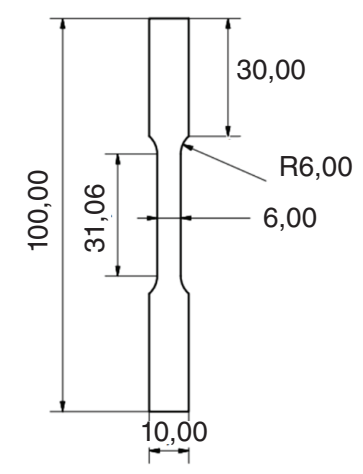

Figura 3: Corpo de prova de tração utilizado (dimensões em mm).

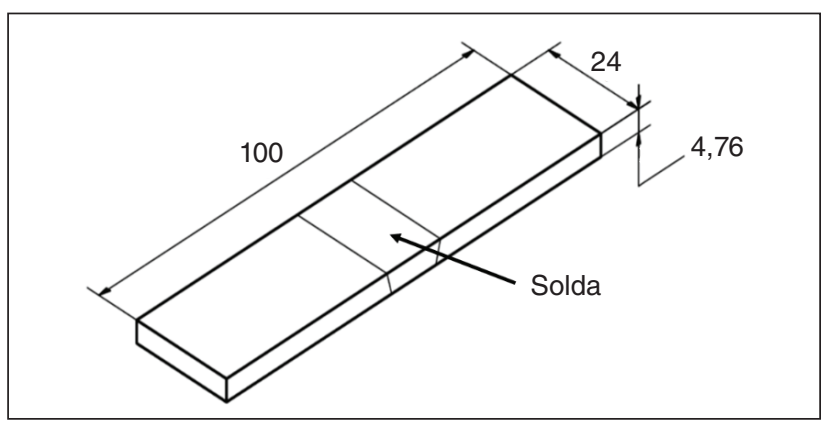

Figura 4: Corpo de prova de dobramento utilizado (dimensões em mm).

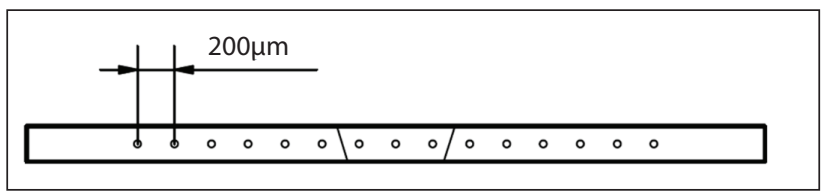

Figura 5: Posição onde foram realizadas as impressões de dureza.
Por fim, a fim de determinar a magnitude das tensões residuais nas juntas soldadas, empregou-se uma variação da técnica semi-destrutiva do furo cego, conforme desenvolvido por Siqueira Filho et al. $^{24}$. O método consiste em utilizar uma máquina de medição de coordenadas e determinar a variação das coordenadas pré-determinadas para o centro de um furo após a realização deste. Espera-se que ocorra variação das coordenadas em função da relaxação das tensões residuais de soldagem. Para tanto, são empregadas as Eqs. 1 e 2:

$$
\begin{gathered}
\sigma_{x}=\frac{E}{1-v^{2}}\left(\varepsilon_{x}+v \varepsilon_{y}\right) \\
\sigma_{y}=\frac{E}{1-v^{2}}\left(\varepsilon_{y}+v \varepsilon_{x}\right)
\end{gathered}
$$

onde: $\sigma_{x}$ é a magnitude da tensão residual longitudinal; $\sigma_{y}$ é a magnitude da tensão residual transversal; E é o módulo de elasticidade; $v$ é o coeficiente de Poisson; $\varepsilon_{x}$ é a deformação característica na direção longitudinal; $\varepsilon_{y}$ é a deformação característica na direção transversal.

As coordenadas de referência foram determinadas em um programa CNC, em código FANUC, e os furos foram realizados utilizando-se um centro de usinagem D-600 da Romi. O esquema da furação é apresentado na Fig. 6. As coordenadas após a realização da furação foram determinadas utilizando-se uma máquina de medição de coordenadas modelo TESAMICROHITE $3 \mathrm{D}$, com resolução de $0,1 \mu \mathrm{m}$.

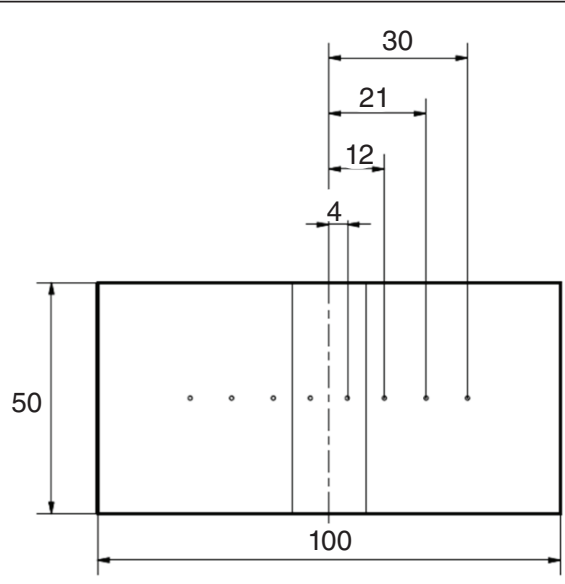

Figura 6: Esquema da furação para aferição da tensão residual (dimensões em mm).

\section{RESULTADOS E DISCUSSÃO}

Inicialmente, foram realizadas inspeções visuais superficiais em todas as juntas soldadas pelas ferramentas de diferentes geometrias a fim de visualizar defeitos superficiais na solda, como falta de penetração, irregularidades, espaços vazios e excesso de rebarba 
(flash), já que os mesmos podem influenciar nos resultados dos ensaios mecânicos posteriores.

Foram visualizados, em algumas juntas soldadas, o furo remanescente (keyhole), que é proveniente do pino da ferramenta e ocorre ao fim do processo FSW, quando a ferramenta é recolhida ${ }^{25}$, como ilustra a Fig. 7. O keyhole é considerado um defeito específico do processo de soldagem por FSW, já que o mesmo é originado pelo pino da ferramenta e pelo excesso de força uniaxial exercida durante o processo.

Foi visualizado em algumas placas soldadas o excesso de rebarba nas laterais da solda, possivelmente oriundo do alto valor da força axial aplicada durante a soldagem. O flash resulta em uma grande perda de material durante o processo, o qual escoa para as laterais da solda após a passagem da ferramenta. Na Fig. 8 é possível visualizar o excesso de rebarba em uma junta soldada.

Defeitos associados a espaços vazios e irregularidades não foram observados visualmente nas juntas. Independentemente do keyhole e flash observados em algumas juntas, os parâmetros do processo FSW foram considerados adequados para a liga em estudo, gerando juntas livres de defeitos superficiais, sem sinal de distorções, já que tanto o keyhole como o flash são considerados defeitos específicos do processo, ocorrendo frequentemente nas soldagens por FSW. A Fig. 9 ilustra uma junta isenta de irregularidades e espaços vazios.

Com relação às propriedades mecânicas, foram analisados primeiramente os perfis de dureza das juntas soldadas ao longo da zona misturada (ZM) e do metal de base (MB). Os resultados plotados apresentam o perfil de dureza, observado na Fig. 10, já na

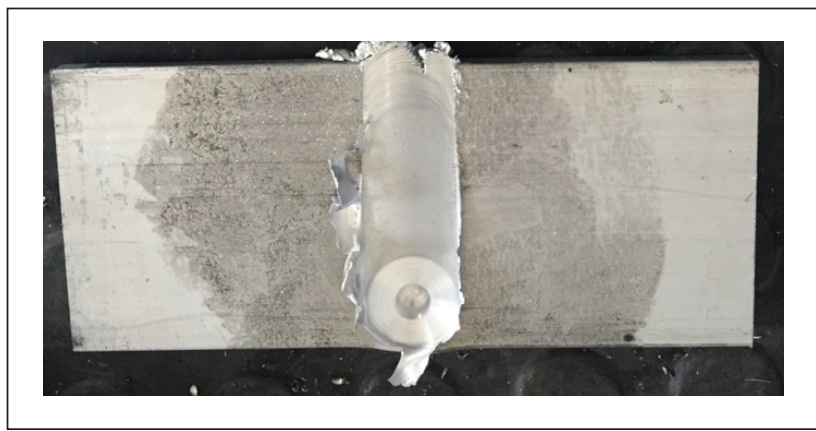

Figura 7: Presença do furo remanescente na junta soldada de alumínio por FSW.

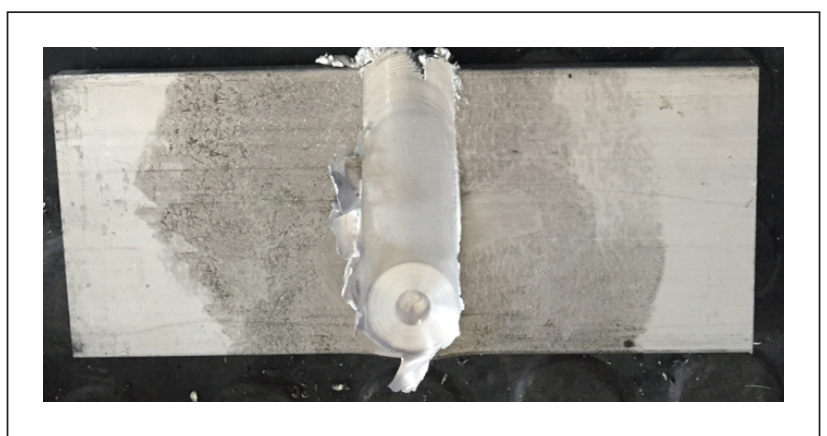

Figura 8: Excesso de rebarba de uma junta soldada de alumínio por FSW.
Fig. 11 é possível observar o boxplot com as medidas de tendência central e dispersão da dureza. É possível inferir que não houve variação significativa da dureza na estrutura, divergindo com os resultados observados em literatura técnica ${ }^{3,26}$.

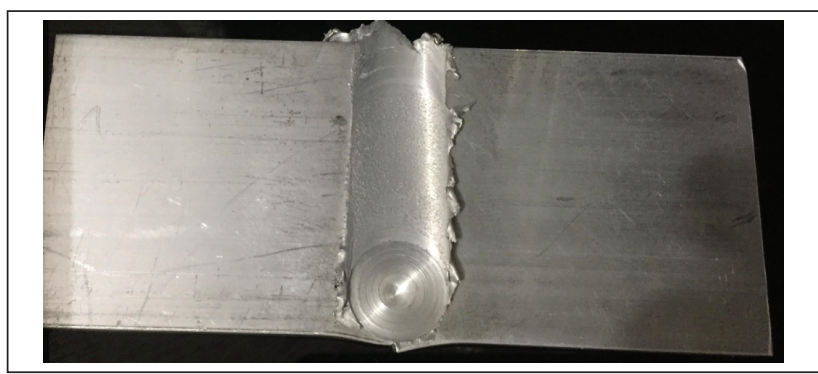

Figura 9: Junta soldada de alumínio por FSW.

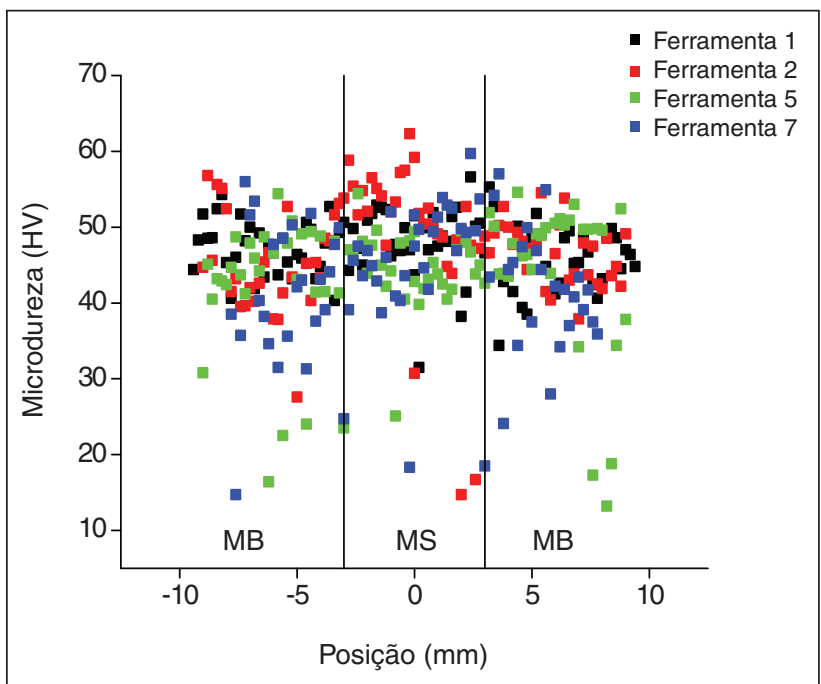

Figura 10: Disposição dos pontos ao longo da junta soldada, onde foi realizado o ensaio de dureza.

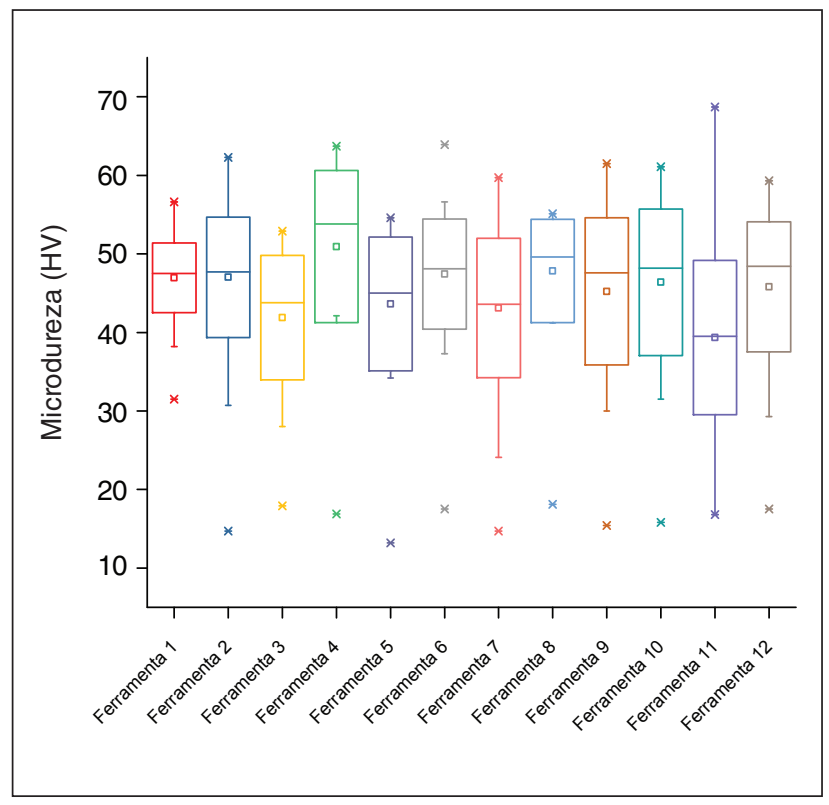

Figura 11: Boxplot dos valores de dureza para as 12 ferramentas avaliadas. 
De acordo com Souza ${ }^{26}$, a dureza na zona misturada tende a aumentar levemente em relação ao metal de base, possivelmente devido à intensa atividade mecânica nessa região, o que faz com que os grãos, antes considerados grosseiros, se tornem mais refinados. Já na zona termomecanicamente afetada e na zona termicamente afetada, regiões adjacentes à zona misturada, o calor não é o suficiente para promover o refinamento dos grãos, podendo ocorrer dissolução dos precipitados formados devido à grande quantidade de energia térmica gerada pela energia mecânica fornecida, fazendo com que ocorra a migração de magnésio do interior dos grãos para formar precipitados de $\mathrm{Mg}_{2} \mathrm{Al}_{3}$, como reportado por Moshwan et al. ${ }^{27}$, promovendo uma leve queda na dureza ${ }^{28}$. Isto não foi verificado nas juntas soldadas em estudo, pois não foi observada variação de dureza, como mostram a Fig. 10 e a Tabela 3. Algumas hipóteses podem ser levantadas a fim de explicar tal acontecimento, como:

- O tratamento térmico de recozimento da liga é necessário antes das operações de conformação mecânica a frio devido à ocorrência de encruamento. Ligas da série 5000, cujo aumento de dureza só pode ser obtido mediante trabalho mecânico, consequentemente encruamento, sofrem influência direta na redução da dureza quando submetidas ao tratamento térmico de recozimento alterando o tamanho de grão, de maneira que as ligas após este tratamento não sejam sensíveis ao trabalho mecânico da ferramenta;

- Baixo nível de encruamento da liga, que não foi o suficiente para afetar a dureza nas regiões da ZTA e ZTMA

- Dissolução dos precipitados que controlam a dureza em determinadas regiões da solda. Embora ocorra o refinamento de grãos na zona misturada, a dureza dessa zona é mais influenciada pela dispersão de vários compostos intermetálicos e dissolução de partículas de segunda fase $\left(\beta-\mathrm{Mg}_{2} \mathrm{Al}_{3}\right)$, o que leva à degradação do desempenho global das juntas soldadas ${ }^{27}$.

Tabela 3: Valores de dureza (HV) da zona misturada e no metal de base.

\begin{tabular}{|c|c|c|c|c|}
$\begin{array}{c}\text { Tipo de } \\
\text { Ferramenta } \\
\text { utilizada }\end{array}$ & \multicolumn{2}{|c|}{ Zona misturada } & \multicolumn{2}{c|}{ Metal de base } \\
\cline { 2 - 5 } & $\boldsymbol{\mu}$ & $\boldsymbol{\sigma}$ & $\boldsymbol{\mu}$ & $\boldsymbol{\Sigma}$ \\
\hline Ferramenta 1 & 43,42 & 8,56 & 46,43 & 3,98 \\
\hline Ferramenta 2 & 51,00 & 14,22 & 45,77 & 5,40 \\
\hline Ferramenta 5 & 45,05 & 4,52 & 43,44 & 5,18 \\
\hline Ferramenta 7 & 41,77 & 15,73 & 41,91 & 7,21 \\
\hline
\end{tabular}

A fim de averiguar a resistência mecânica das juntas soldadas obtidas por diferentes perfis de pino da ferramenta, foram realizados ensaios de tração uniaxial. A coletânea desses ensaios pode ser observada na Fig. 12.
Foi observado, pela Fig. 12, que as juntas soldadas suportaram grandes deformações, acima de $12 \%$, o que condiz com a literatura ${ }^{3}$. De acordo com Capelari e Mazzaferro ${ }^{3}$, que analisaram três tipos de geometrias de pinos, cônica lisa, facetada e cônica com rosca, os resultados foram semelhantes entre as três geometrias, como o realizado nesta pesquisa, suportando valores de deformações em torno de $20 \%$.

Os valores de limite de escoamento (LE), limite de resistência à tração (LRT), e deformação de fratura (DEF) podem ser observados na Fig. 13. É possível notar uma redução do limite de escoamento e do limite de resistência à tração em relação ao metal de base, resultados já esperados, visto que devido ao aporte de calor gerado pela velocidade relativa entre a ferramenta rotativa $e$ o substrato há um aumento do crescimento de grãos e dissolução de partículas de segunda fase na zona soldada ${ }^{29}$. Já em relação às comparações entre as geometrias da ferramenta, desconsiderando o metal de base, foi notada invariabilidade dos parâmetros, conforme observado por Capelari ${ }^{3}$, quando comparou três tipos

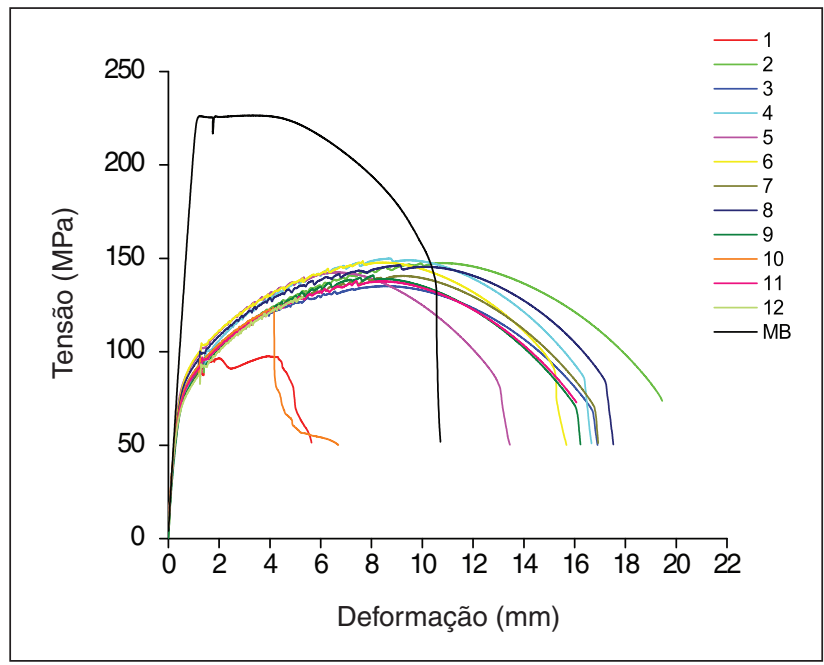

Figura 12: Curva tensão $\times$ deformação para as juntas soldadas de alumínio pelo processo FSW e do MB.

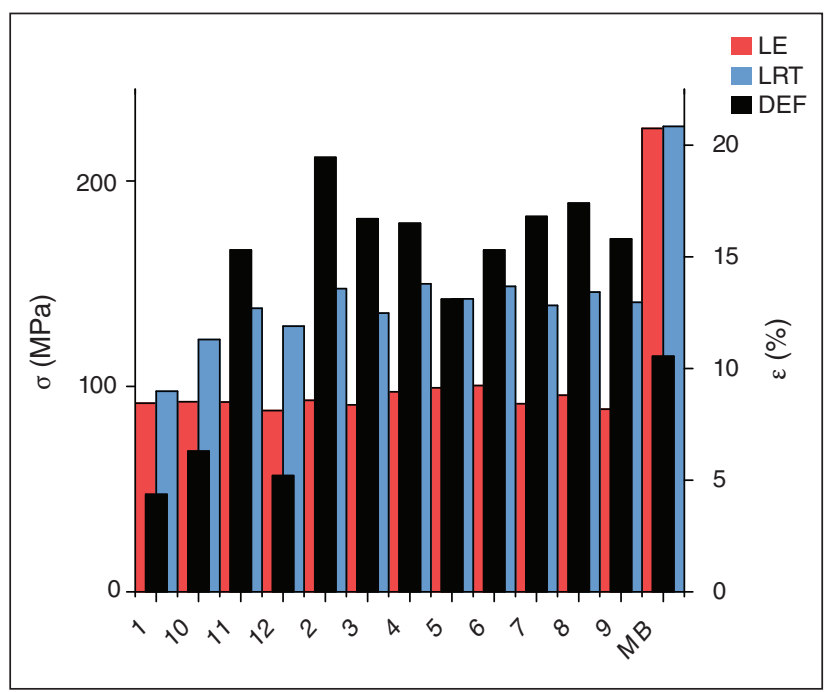

Figura 13: Principais valores obtidos no ensaio de tração uniaxial. 
de geometrias de pinos, cônica lisa, facetada e cônica com rosca. Os menores valores encontrados foram os das ferramentas 1 e 10 , apresentando limites inferiores aos demais, conforme pode ser visto na Fig. 13.

A fim de qualificar a ductilidade das juntas soldadas foram realizados ensaios de dobramento a $60^{\circ}$. Na Fig. 14 é possível observar duas juntas soldadas submetidas a este ensaio. A Fig. 15 ilustra o gráfico de carga aplicada durante o ensaio versus o deslocamento atingido. Dentre as 12 ferramentas, apenas a da geometria de pino 12 obteve um menor deslocamento.

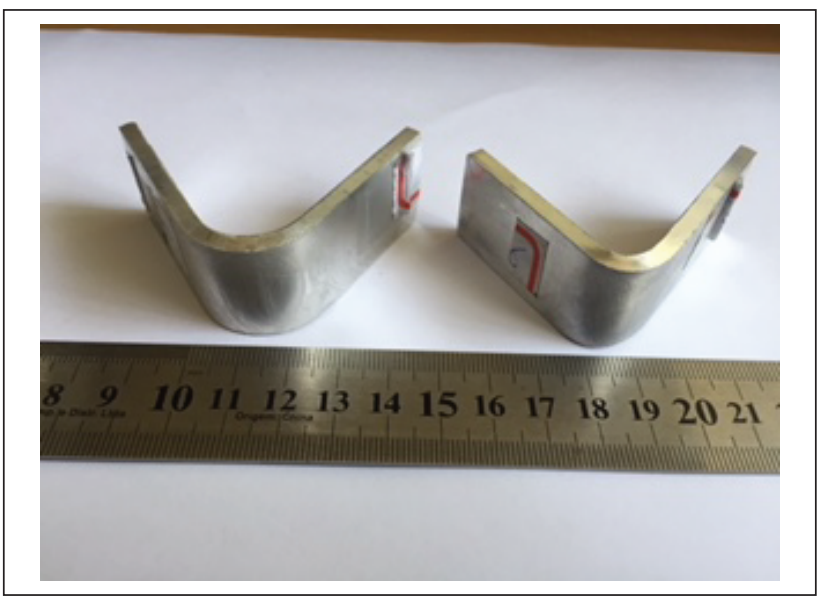

Figura 14: Junta soldada após o ensaio de dobramento a $60^{\circ}$.

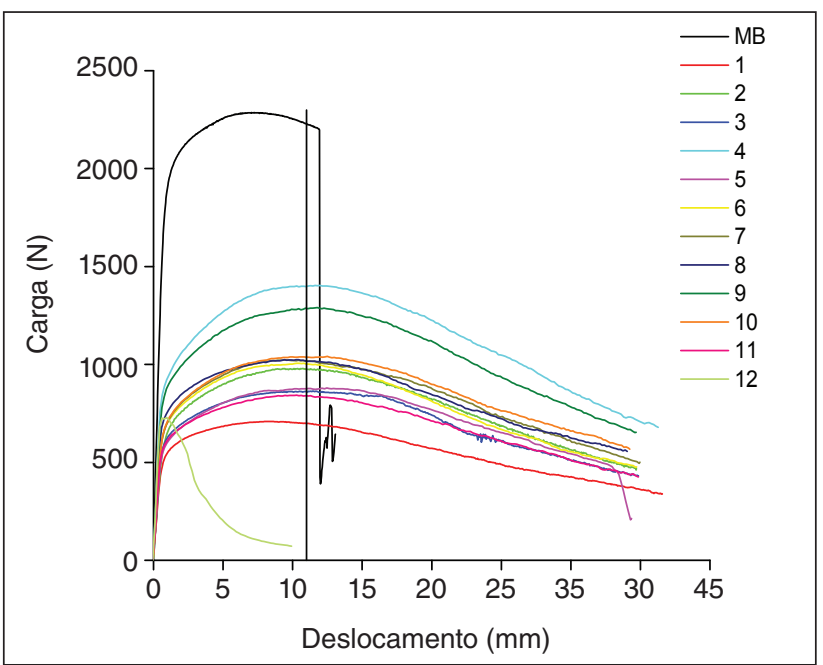

Figura 15: Curva carga versus deslocamento do ensaio de dobramento para as juntas soldadas de alumínio pelo processo FSW e do MB.

A Tabela 4 contém um resumo da carga máxima suportada nos ensaios de dobramento para as 12 juntas soldadas com diferentes ferramentas. Dentre as ferramentas analisadas, a geometria do pino da ferramenta 1 apresentou menor carga suportada, seguida da ferramenta 12 . De acordo com resultados anteriores, a ferramenta 1 já apresentava uma menor resistência, se comparada às demais, quando analisada pelo ensaio de tração uniaxial. Já a ferramenta 12 , apesar de apresentar uma menor carga máxima suportada no ensaio de dobramento, obteve um alto desvio padrão.
Tabela 4: Carga máxima suportada no ensaio de dobramento para as juntas soldadas pelas 12 ferramentas diferentes.

\begin{tabular}{|c|c|c|}
\hline Material & $\begin{array}{l}\text { Carga máxima média } \\
\text { suportada (N) }\end{array}$ & $\begin{array}{l}\text { Desvio padrão } \\
\text { (N) }\end{array}$ \\
\hline Metal de Base & 2290 & 22 \\
\hline $\begin{array}{c}\text { Junta Soldada pela } \\
\text { Ferramenta } 1\end{array}$ & 699 & 26 \\
\hline $\begin{array}{c}\text { Junta Soldada pela } \\
\text { Ferramenta } 2\end{array}$ & 966 & 39 \\
\hline $\begin{array}{c}\text { Junta Soldada pela } \\
\text { Ferramenta } 3\end{array}$ & 858 & 26 \\
\hline $\begin{array}{c}\text { Junta Soldada pela } \\
\text { Ferramenta } 4\end{array}$ & 1400 & 25 \\
\hline $\begin{array}{l}\text { Junta Soldada pela } \\
\text { Ferramenta } 5\end{array}$ & 881 & 44 \\
\hline $\begin{array}{l}\text { Junta Soldada pela } \\
\text { Ferramenta } 6\end{array}$ & 1002 & 40 \\
\hline $\begin{array}{l}\text { Junta Soldada pela } \\
\text { Ferramenta } 7\end{array}$ & 1013 & 62 \\
\hline $\begin{array}{c}\text { Junta Soldada pela } \\
\text { Ferramenta } 8\end{array}$ & 1024 & 75 \\
\hline $\begin{array}{l}\text { Junta Soldada pela } \\
\text { Ferramenta } 9\end{array}$ & 1288 & 47 \\
\hline $\begin{array}{c}\text { Junta Soldada pela } \\
\text { Ferramenta } 10\end{array}$ & 1036 & 60 \\
\hline $\begin{array}{c}\text { Junta Soldada pela } \\
\text { Ferramenta } 11\end{array}$ & 836 & 64 \\
\hline $\begin{array}{c}\text { Junta Soldada pela } \\
\text { Ferramenta } 12\end{array}$ & 724 & 71 \\
\hline
\end{tabular}

Comparando os valores obtidos do ensaio de dobramento juntamente com o de tração, pode-se observar que as ferramentas 4 e 9 apresentaram melhores resultados de resistência mecânica. Essas duas ferramentas possuem geometria do pino cilíndrica, diferindo entre si apenas pela existência de rosca ou não, como pode ser visto na Fig. 16. Essas duas geometrias podem ter proporcionado melhores propriedades mecânicas devido à maior uniformidade do fluxo de metal na zona misturada, já que ferramentas de perfil simplesmente cilíndrico proporcionam maior uniformidade na velocidade de escoamento do material ${ }^{30,31}$. Já as ferramentas de soldagem 1, 10 e 12 possivelmente apresentaram piores propriedades mecânicas devido a sua geometria não axissimétrica, conforme Fig. 17, resultando em uma distribuição não uniforme do fluxo de metal, já que a velocidade do fluxo de metal na ponta do pino da ferramenta será maior que na base do pino $^{29,30}$.

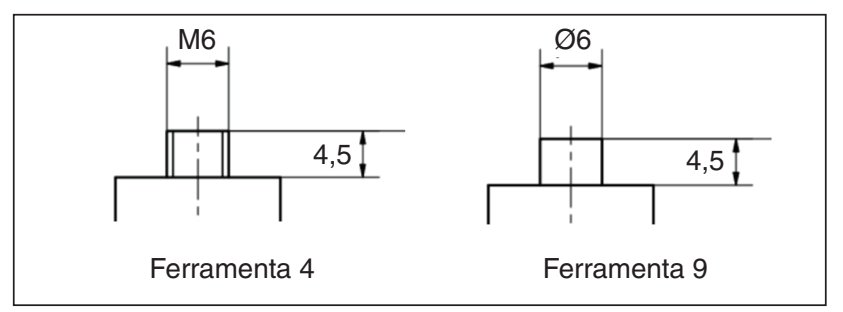

Figura 16: Perfil dos pinos de ferramentas de soldagem em estudo que apresentaram as melhores propriedades mecânicas. 


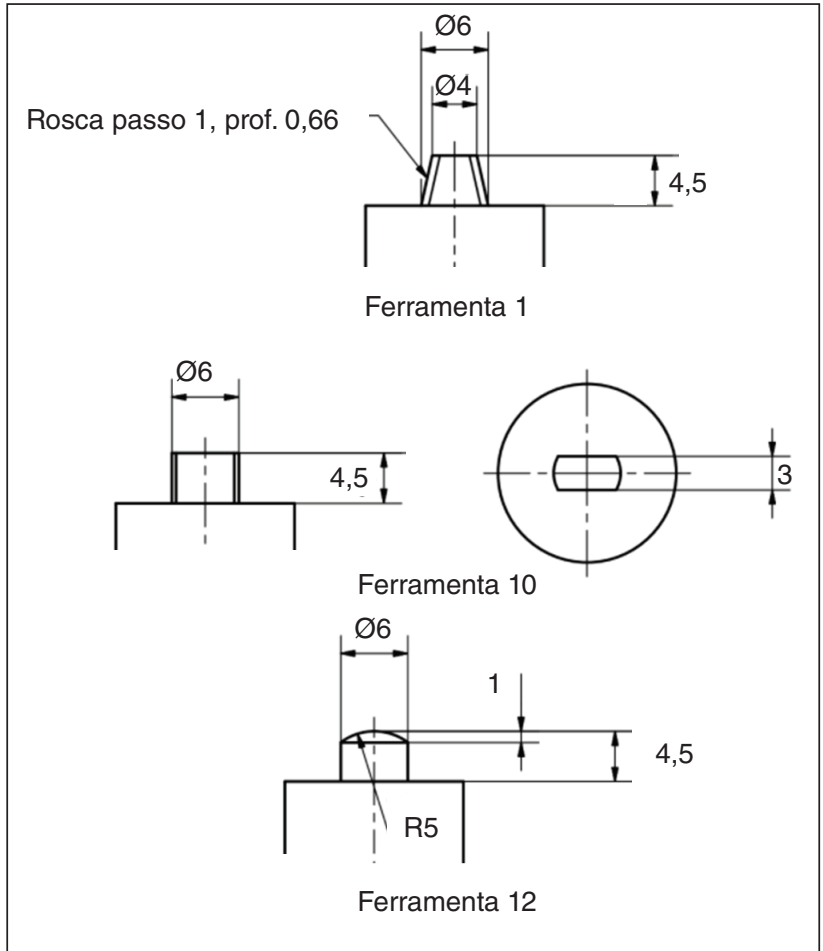

Figura 17: Perfil dos pinos de ferramentas de soldagem em estudo que apresentaram as piores propriedades mecânicas.

As tensões residuais são geradas como consequência da soldagem e podem prejudicar/comprometer o comportamento da junta no que diz respeito à resistência mecânica e resistência à corrosão ${ }^{13}$. A principal fonte de tensões residuais em juntas soldadas é a contração no resfriamento de regiões diferentemente aquecidas e plastificadas, já que a temperatura no processo é suficiente para que o módulo de elasticidade e a resistência ao escoamento do metal sejam reduzidos. Essa redução do limite de escoamento pode ser visualizada na Fig. 13, em que, para os materiais soldados, essa resistência diminuiu cerca de $50 \%$.

A Fig. 18 ilustra os perfis de tensões residuais longitudinais e transversais. Através das tensões longitudinais é possível observar a assimetria do processo de soldagem por FSW, já que é visível a diferença dos perfis no lado esquerdo e direito. Essa assimetria é típica do processo de soldagem por $\mathrm{FSW}^{26}$.

De acordo com Souza et al. $^{26}$, a tensão residual longitudinal é sempre de natureza trativa no interior da zona de mistura e, afastando-se das extremidades da zona de mistura, em direção às ZTAs, as tensões residuais trativas diminuem e tensões compressivas surgem. Esse perfil só foi visualizado nas juntas soldadas pela ferramenta 1 , as demais ferramentas apresentaram invariabilidade nos pontos medidos. Essa uniformidade pode ser explicada pela uniformidade da temperatura fornecida pelo atrito entre o ombro da ferramenta e as chapas, que não forneceram variações bruscas a ponto de alterar os perfis de tensões residuais. Além disso, as tensões residuais longitudinais têm magnitude máxima da ordem de $50 \mathrm{MPa}$, com exceção das juntas soldadas pelas ferramentas $1,4,8$ e 9 que apresentaram tensões residuais

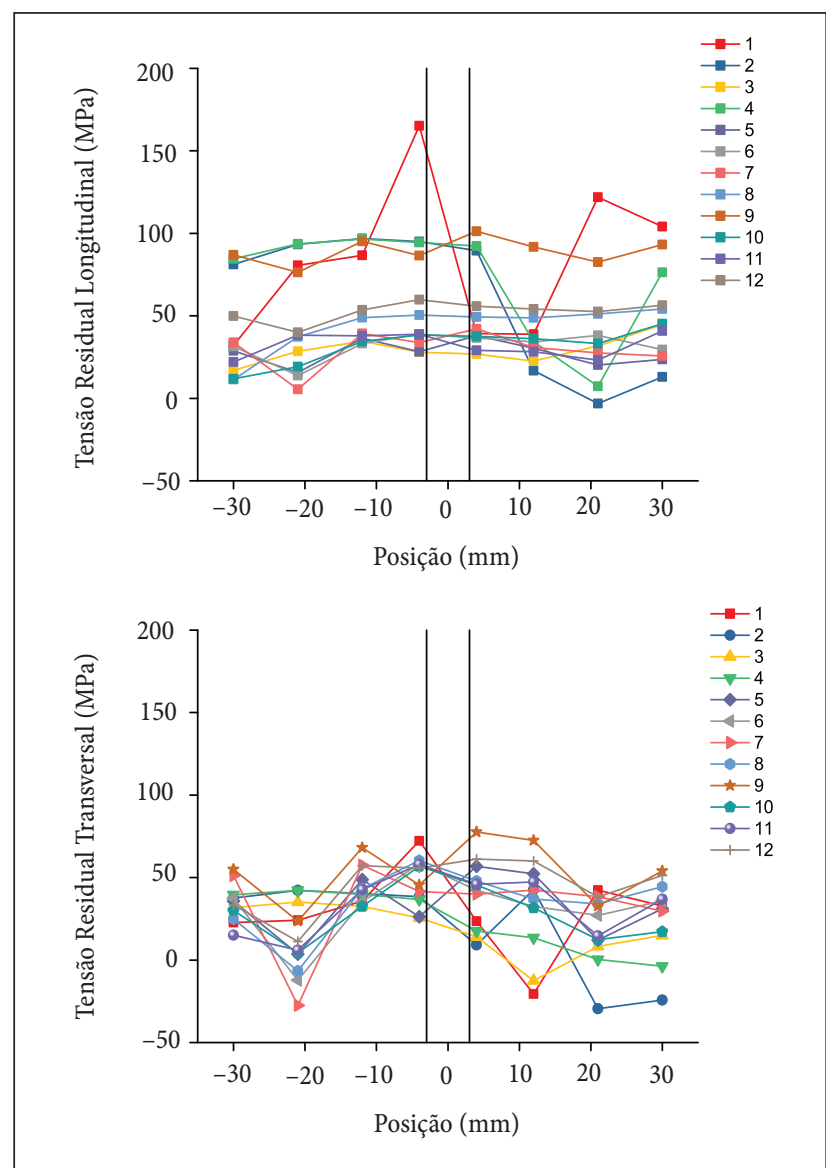

Figura 18: Magnitude das tensões residuais longitudinais e transversais nas juntas soldadas.

entre 75 e $100 \mathrm{MPa}$ no lado de avanço da junta, praticamente o limite de escoamento (LE) observado para todas as condições em estudo. O perfil e a magnitude das tensões residuais observados é similar ao obtido por Zapata et al. ${ }^{32}$. Além disso, a magnitude das tensões residuais é bem menor que aqueles geralmente observados por processos convencionais de soldagem ao arco elétrico, que varia entre 200 e $250 \mathrm{MPa}$, conforme indicado por Bajpei et al. ${ }^{33}$

Vale destacar que a junta soldada pela ferramenta 1 apresentou tensões residuais de magnitude superior ao LE, podendo ser um forte indicativo para o baixo desempenho nos ensaios mecânicos das juntas soldadas por esta ferramenta.

\section{CONCLUSÕES}

Não foram detectados defeitos de falta de penetração, irregularidades e espaços vazios, indicando que os parâmetros do processo FSW foram considerados adequados para a liga em estudo.

Em relação à dureza, foi visto que não houve variação na estrutura, divergindo com os resultados observados em literatura técnica, sendo necessária a realização da análise metalográfica para comprovar algumas hipóteses levantadas.

No ensaio de tração foi possível notar uma redução do limite de escoamento e do limite de resistência à tração em relação ao metal 
de base, resultados já esperados de acordo com a bibliografia. Em relação às comparações entre as geometrias da ferramenta, foi notada uma invariabilidade nos valores de limite de escoamento e limite de resistência à tração. Os menores valores encontrados foram das ferramentas 1 e 10 .

Comparando os valores obtidos do ensaio de dobramento juntamente com o de tração, foi possível observar que as ferramentas 4 e 9 apresentaram melhores resultados de resistência mecânica, se comparadas às demais.

Em relação aos perfis de tensões residuais, foi constatado que as ferramentas apresentaram invariabilidade nos pontos medidos. Essa uniformidade pode ser explicada pela uniformidade da temperatura durante o processo.

\section{AGRADECIMENTOS}

Os autores agradecem à PRPIPG do Instituto Federal de Educação, Ciência e Tecnologia da Paraíba pelo fomento e bolsa, por meio do edital INTERCONECTA 2017.

\section{REFERÊNCIAS}

1. ABAL. Ligas. [Internet]. São Paulo: Associação Brasileira do Alumínio. [Acesso 2016 novembro 30] Disponível em: http:// abal.org.br/aluminio/caracteristicas-quimicas-e-fisicas/ligas/

2. Ferrari R. Caracterização de liga metálica a base de alumínio magnésio obtida por método convencional após tratamentos termomecânicos visando estruturas microcristalinas [Dissertação de Mestrado]. São Paulo: Universidade de São Paulo; 2008.

3. Capelari TV, Mazzaferro JAE. Avaliação da Geometria de Ferramenta e Parâmetros do Processo FSW na Soldagem da Liga de Alumínio AA 5052. Soldagem Insp. São Paulo. 2015;14(3):215-227

4. Coraini R, Kobayashi Y. Soldagem GMAW Robotizada de Alumínio: influência do tipo de chanfro, tecimento e sentido de laminação na distorção angular. In: III Seminário da Pósgraduação em Engenharia Mecânica; Bauru, Brasil; 2009.

5. Lee CY, Lee WB, Yeon YM, Jung SB. The joint characteristics of friction stir welding Mg-Zn-Y alloy. Materials Science Forum. 2005;475-479:555-558. Disponível em: https://doi. org/10.4028/www.scientific.net/MSF.475-479.555

6. Souza GS. Influência da geometria da ferramenta na soldagem da liga de alumínio 5052 pelo processo friction stir welding [Dissertação de Mestrado]. Curitiba: UTFPR; 2010.

7. Mishra RS. Friction stir processing technologies. Advanced Materials \& Process. 2003;161(10):43-46.

8. Pao PS, Gill SJ, Feng CR, Sankaran KK. Corrosion-fatigue crack growth in friction stir welded Al 7050. Scripta Materialia. 2001;45(5):605-612. Disponível em: https://doi.org/10.1016/ S1359-6462(01)01070-3

9. Sutton MA, Yang B, Reynolds AP, Taylor R. Microstructural studies of friction stir welds in 2024-T3 aluminum. Materials Science and Engineering A. 2002;323(1-2):160166. Disponível em: https://doi.org/10.1016/S09215093(01)01358-2
10. Williams SW. Welding of Airframes using Friction Stir. Air \& Space Europe. 2001;3(3-4):64-66. Disponível em: https://doi. org/10.1016/S1290-0958(01)90059-0

11. ASM International. Metals Handbook. Properties and selection: nonferrous alloys and special purpose materials. ASM International; 1990

12. Zhao $Y$, Lin $S$, Wu L, Qu F. The influence of pin geometry on bonding and mechanical properties in friction stir weld 2014 Al alloy. Materials Letters. 2005;59(23):2948-2952. Disponível em: https://doi.org/10.1016/j.matlet.2005.04.048

13. Cofiñe RC. Análise das tensões residuais em uma junta soldada em condições overmatch [Trabalho de Conclusão de Curso]. São Bernardo do Campo: FEl; 2010.

14. Modenesi P. Efeitos mecânicos do ciclo térmico [Dissertação de Mestrado]. Belo Horizonte: Universidade Federal de Minas Gerais; 2008.

15. Thomas WM, Nicholas ED, Needham JC, Murch MG, TempleSmith P, Dawes CJ, inventores; Welding Institute England. Friction stir butt welding. International Patent no. 9125978.8. 1991.

16. Boz, M.; Kurt, A. The influence of stirrer geometry on bonding and mechanical properties in friction stir welding process. Materials and Design. 2004;25(4):343-347. Disponível em: https://doi.org/10.1016/j.matdes.2003.11.005

17. Buffa G, Hua J, Shivpuri R, Fratini L. Design of the friction stir welding tool using the continuum based FEM model. Materials Science and Engineering A. 2006;419(1-2):381-388. Disponível em: https://doi.org/10.1016/j.msea.2005.09.041

18. Poetscher F, Paiva FGA, Vilaça PJ, Brandi SD. Caracterização de Junta Soldada por FSW de Alumínio AA6063 T6. In: V Congresso Brasileiro de Engenharia De Fabricação; Belo Horizonte, Brasil; 2009.

19. Osinski G, Camargo JF, Lermen RT. Influência da geometria da ferramenta e parâmetros na soldagem de chapas sobrepostas de alumínio pelo processo friction stir welding. In: $3^{\text {a }}$ SIEF. Horizontina, Brasil; 2013

20. Fioravanti AS. Soldagem por FSW de ligas de alumínio ALCLAD AA2024-T3 e AA7075-T6 [Dissertação de Mestrado]. Porto Alegre: Universidade Federal do Rio Grande do Sul; 2008.

21. Cerveira RLLP. Caracterização experimental do comportamento mecânico sob solicitação multiaxial em junções de chapas AA2024-T3 soldadas por fricção-mistura (FSW) [Dissertação de Mestrado]. São Paulo: Universidade de São Paulo; 2008.

22. Capelari TV. Avaliação da geometria de ferramenta e parâmetros do processo FSW na soldagem da liga de alumínio AA 5052 [Dissertação de Mestrado]. Porto Alegre: Universidade Federal do Rio Grande do Sul; 2006

23. Gipiela ML, Martins F. Influência dos parâmetros de processo na soldagem da liga de alumínio 5052 pelo processo FSW. In: $8^{\circ}$ Congresso Brasileiro de Engenharia de Fabricação; Salvador, Brasil; 2015

24. Siqueira Filho AV, Rolim TL, Yadava YP, Cardoso FIB, Guimarâes PB, Maciel, TM, et al. Development of methodology for measurements of residual stresses in welded joint based on displacement of points in a coordinated table. Materials Research. 2013;16(2):322-326. Disponível em: http://dx.doi. org/10.1590/S1516-14392013005000001 
25. Carvalho JM. Análise estática e de fadiga de uniões soldadas por friction stir welding [Dissertação de Mestrado]. Campinas: Universidade Estadual de Campinas; 2012

26. Souza DM, Pinto HC, Santos JF, Coelho RS. Estudo das propriedades mecânicas, microestruturais e tensões residuais da liga de alumínio AA 5083-H111 soldadas por friction stir welding - FSW. In: $8^{\circ}$ Congresso Brasileiro de Engenharia de Fabricação; Salvador, Brasil; 2015.

27. Moshwan R, Yosof F, Hassan MA, Rammat SM. Effect of tool rotational speed on force generation, microstructure and mechanical properties of friction stir welded $\mathrm{Al}-\mathrm{Mg}-\mathrm{Cr}-\mathrm{Mn}$ (AA 5052-O) alloy. Materials and Design. 2015;66(A):118-128. Disponível em: https://doi.org/10.1016/j.matdes.2014.10.043

28. Tiwari SK, Shukla DK, Chandra R. Friction stir welding of aluminum alloys: a review. International Journal of Mechanical, Aerospace, Industrial and Mechatronics Engineering. 2013;7(12):2403-2408.

29. Ilangovan M, Boopathy SR, Balasubramanian V. Effect of tool pin profile on microstructure and tensile properties of friction stir welded dissimilar AA 6061-AA 5086 aluminium alloy joints. Defence Technology. 2015;11(2):174-184. Disponível em: https://doi.org/10.1016/j.dt.2015.01.004

30. Ji S, Jin $Y$, Yue $Y$, Zhang L, Lv Z. The effect of tool geometry on material flow behavior of friction stir welding of titanium alloy. Engineering Review. 2013;33(2):107-113

31. Mehta KP, Badheka VJ. Effects of tool pin design on formation of defects in dissimilar friction stir welding. Procedia Technology. 2016;23:513-518. Disponível em: https://doi. org/10.1016/j.protcy.2016.03.057

32. Zapata J, Toro M, López D. Residual stresses in friction stir dissimilar welding of aluminum alloys. Journal of Materials Processing Technology. 2016;229:121-127. Disponível em: https://doi.org/10.1016/j.jmatprotec.2015.08.026

33. Bajpei T, Chelladurai H, Ansari MZ. Mitigation of residual stresses and distortions in thin aluminium alloy GMAW plates using different heat sink models. Journal of Manufacturing Processes. 2016;22:199-210. Disponível em: https://doi. org/10.1016/j.jmapro.2016.03.011 